\title{
O desafio do cuidado do enfermeiro ao paciente com lesão musculoesquelética que vivencia a COVID-19: relato de experiência
}

The challenge of nursing care to patients with musculoskeletal injury who experience COVID-19: experience report

El desafio de la atención de enfermería al paciente con lesión musculoesquelética que vive COVID19: informe de experiência

Letícia Aparecida Marincolo Domenis ORCID: https://orcid.org/0000-0003-4629-000X Universidade Federal Fluminense, Brasil E-mail:leticiamarincolo@gmail.com Donizete Vago Daher ORCID: https://orcid.org/0000-0001-6249-0808 Universidade Federal Fluminense, Brasil E-mail:donidaher@gmail.com

Bárbara Stohler Sabença de Almeida ORCID: https://orcid.org/0000-0001-7274-2925 Universidade Federal Fluminense, Brasil E-mail:babistohler@gmail.com

Maryanne Carvalho Araújo ORCID: https://orcid.org/0000-0001-9557-4231 Universidade do Estado do Rio de Janeiro, Brasil E-mail:mca.maryanne@gmail.com Juliane de Macedo Antunes ORCID: https://orcid.org/0000-0002-97638291 Universidade Federal Fluminense, Brasil E-mail:julianedemacedoantunes@hotmail.com Maria Fernanda Muniz Ferrari ORCID: https://orcid.org/0000-0001-6606-8938 Universidade Federal Fluminense, Brasil E-mail:mfmferrari@gmail.com

\section{Resumo}

Objetivo: Relatar sobre o cuidado do enfermeiro ao paciente com lesão musculoesquelética que vivencia a COVID19. Metodologia: Abordagem qualitativa descritiva do tipo relato de experiência ocorrido entre os meses de março a julho de 2020, em um instituto nacional de referência para tratamento de Traumatologia e Ortopedia, localizado na cidade do Rio de Janeiro. Resultados e Discussão: A experiência pôde ser narrada por meio de três temas: Fluxo de atendimento e o perfil de pacientes atendidos; Complexidade do atendimento ao paciente com lesão musculoesquelética que vivencia a COVID-19; Novos desafios para a enfermagem ortopédica gerados pela pandemia da COVID-19. Conclusão: Na perspectiva da singularidade do cuidado, considerou-se complexo e desafiador cuidar dos pacientes que vivenciam, duplamente, a lesão musculoesquelética e a COVID-19. Uma assistência de enfermagem especializada durante o tratamento mostrou-se imprescindível à medida que a ação do enfermeiro gera um forte elo com os pacientes, reforçando a adesão ao tratamento e contribuindo na redução dos quadros de complicações decorrentes da infecção por coronavírus.

Palavras-chave: Enfermeiras e enfermeiros; Enfermagem ortopédica; Coronavírus, COVID-19; Cuidados de enfermagem.

\footnotetext{
Abstract

Objective: Report on the nurse's care for patients with musculoskeletal injuries who experience COVID-19. Methodology: Qualitative descriptive approach of the type of experience report that occurred between the months of March and July of 2020, in a national institute of reference for traumatology and orthopedics treatment, located in the city of Rio de Janeiro. Results and discussion: The experience could be narrated through three themes: Flow of caring and the profile of patients seen; Complexity of the care of patients with musculoskeletal injuries who experience COVID-19; New challenges for orthopedic nursing generated by the COVID-19 pandemic. Final considerations: From the perspective of the singularity of care, it was considered complex and challenging to care of the patients who
} 
experience, doubly, the musculoskeletal injury and COVID-19. A specialized nursing care during treatment proved to be indispensable as the nurse's action generates a strong bond with the patients, reinforcing compliance to treatment and contributing to the reduction of complications due to coronavirus infection.

Keywords: Nurses; Orthopedic nursing; Coronavírus; COVID-19; Nursing care.

\section{Resumen}

Objetivo: Informar sobre la atención de enfermería a pacientes con lesiones musculoesqueléticas que padecen COVID-19. Metodología: Enfoque descriptiva cualitativa del tipo de experiencia ocurrida entre los meses de marzo a julio de 2020, en un instituto nacional de referencia para el tratamiento de Traumatología y Ortopedia, ubicada en la ciudad de Rio de Janeiro. Resultados y Discusión: La experiencia se pudo contar a través de tres temas: Flujo de atención y perfil de pacientes atendidos; Complejidad de la atención para pacientes con lesiones musculoesqueléticas que experimentan COVID-19; Nuevos desafíos para la enfermería ortopédica generados por la pandemia de COVID19. Consideraciones finales: En la perspectiva de la singularidad del cuidado, se consideró complejo y desafiante atender a los pacientes que experimentan, en dos ocasiones, lesión musculoesquelética y COVID-19. Una atención de enfermería especializada durante el tratamiento resultó fundamental ya que la actuación de la enfermera genera un fuerte vínculo con los pacientes, reforzando la adherencia al tratamiento y contribuyendo a la reducción de las complicaciones derivadas de la infección por coronavirus.

Palabras clave: Enfermeros; Enfermería ortopédica; Coronavírus; COVID-19; Atención de enfermería.

\section{Introdução}

O ano de 2020, com o surgimento da pandemia de COVID-19 causada pelo vírus SARS- CoV-2, representou um dos maiores desafios da história da humanidade. Originada em Wuhan, na China, em dezembro de 2019, esta doença vem ocasionando milhões de infectados em todos os continentes e determinando efeitos devastadores tanto na área da saúde como na economia global (Neto \& Queiroz, 2020).

Como resposta ao surgimento da COVID-19, e com o reconhecimento da rápida disseminação global, em 11 de março de 2020, a Organização Mundial da Saúde (OMS) caracterizou oficialmente o fenômeno como pandemia, e solicitou ação internacional urgente em quatro áreas principais: preparar, detectar, proteger e tratar. O objetivo era tentar reduzir a transmissão, e para tal era fundamental inovar e aprender cada vez mais sobre a COVID-19. As estratégias deveriam, então, estar focadas nos esforços para reduzir a incidência, morbidade e mortalidade, quebrando a cadeia de transmissão humana através do distanciamento social e instituição de quarentena, a qual foi imposta pelas autoridades de cada nação (WHO, 2020).

A detecção rápida da COVID-19 é fundamental para a ruptura da cadeia de disseminação, sendo imprescindível o diagnóstico precoce e fidedigno. A coleta de materiais respiratórios (aspiração de vias aéreas ou indução de escarro) permite a identificação do vírus através do genoma viral (sequenciamento parcial ou total e detecção do RNA viral) e da técnica de proteína C reativa em tempo real, é o exame diagnóstico de escolha (Ministério da Saúde do Brasil, 2020).

A infecção apresenta quadro clínico amplo que pode ir de um resfriado simples a pneumonia grave. A princípio, apresenta características de síndrome gripal cursando com problemas respiratórios leves e febre persistente. Sinais e sintomas aparecem em média de 5 a 6 dias (este intervalo pode ser de 1 a 14 dias) após a infecção e afetam parte dos indivíduos. Os principais são: febre, tosse, dispneia, mialgia, confusão mental, cefaleia, dor de garganta, rinorreia, dor torácica, diarreia e náuseas, vômitos; além de anosmia e ageusia. Os casos leves podem ser tratados em domicílio com instituição das medidas de precaução e acompanhamento na atenção básica, enquanto os graves devem ser encaminhados a um hospital de referência para isolamento e tratamento (Ministério da Saúde do Brasil, 2020).

Dentre essas várias manifestações clínicas, as pulmonares têm importante repercussão que varia de forma leve a grave, com evolução rápida e fulminante. Nos casos moderados e graves estudos mostram a presença de imagem em "vidro fosco", assim um consenso internacional estabeleceu o uso da radiografia de tórax e da tomografia para auxiliar nas análises das possíveis complicações da doença (Rubin, et al., 2020).

O sistema de saúde do Brasil teve que se adaptar a esta nova e complexa realidade que se soma ao atendimento já difícil e precário vigente nas unidades básicas de saúde e nas grandes emergências. Nestas a assistência é voltada aos pacientes 
vítimas de agravos provocados por causas externas, que englobam a violência e acidentes nos grandes centros urbanos; e seu funcionamento transcende a capacidade máxima. O que pode ser observado pela taxa de ocupação acima de 100\%, número insuficiente de profissionais, demanda excessiva de trabalho. A essas limitações acrescenta-se o déficit estrutural e assistencial da rede (ambientes pequenos e com poucos recursos de ventilação, reduzido número de profissionais) (O’Dwyer, Oliveira \& Seta, 2009). É necessário ampliar a oferta de leitos e de profissionais qualificados para o atendimento de pacientes vítimas de traumas por acidentes com segurança e não os expondo e seus familiares ao vírus SARS- CoV-2.

Os acidentes são a oitava causa de morte no mundo e custam cerca de $1 \%$ a 5\% do Produto Interno Bruto (PIB) nos países em desenvolvimento, impactam diretamente os grupos etários economicamente ativos e resultam em 78,2 milhões de lesões não fatais que justificam cuidados médicos. Estas englobam majoritariamente as fraturas (um quarto no membro inferior), seguidas por feridas abertas, lesões superficiais e traumatismo craniano (The World Bank, 2014).

Neste contexto, é fundamental a instituição de um tratamento rápido e eficaz, que minimize a exposição deste paciente ortopédico ao risco de contaminação por SARS- CoV-2. Cabe à equipe de saúde e, em particular ao enfermeiro, organizar e assistir as necessidades desse cliente. Assim, este artigo relata o cuidado realizado pelo enfermeiro ao paciente com lesão musculoesquelética internado em enfermarias de isolamento para COVID-19 em pré ou pós operatório de cirurgia ortopédica. O objetivo é relatar sobre o cuidado do enfermeiro ao paciente com lesão musculoesquelética que vivencia a COVID-19. E se justifica pela singularidade do momento pandêmico atual, no qual todos os profissionais e as instituições de saúde tiveram que se adaptar rapidamente à nova realidade.

\section{Metodologia}

Estudo descritivo, com abordagem qualitativa do tipo relato de experiência, ocorrido entre os meses de março a julho de 2020, tendo como cenário um instituto nacional de referência para tratamento de Traumatologia e Ortopedia, localizado na cidade do Rio de Janeiro. Especificamente, nas enfermarias de isolamento para pacientes infectados ou com suspeita de COVID-19, com aproximadamente 34 leitos, sendo quartos duplos ou individuais, cuja taxa de ocupação oscilou de acordo com o desfecho de cada paciente: admissão, alta ou transferências internas (semi-intensiva ou Centro de Terapia Intensiva).

Esta instituição tem como propósito promover atendimento amplo e específico a pacientes ortopédicos encaminhados pelo Sistema Estadual de Regulação (SER) vigente no estado do Rio de Janeiro. Durante este período, na tentativa de corroborar com a Secretaria Estadual de Saúde - RJ foi realizado um acordo para receber pacientes internados que necessitavam de cirurgias ortopédicas para que em outras instituições fossem reservados leitos exclusivos para COVID-19. No entanto, os pacientes transferidos para o Instituto, em sua maioria apresentaram sintomas dias depois da internação e após confirmação receberam, além de cuidados ortopédicos, cuidados para a COVID-19.

Com isso a questão de pesquisa que orientou a experiência foi: Como se efetivou o cuidado do enfermeiro ao paciente com lesão musculoesquelética e que vivencia a COVID-19 em uma enfermaria de isolamento?

Uma pesquisa de abordagem qualitativa, utiliza as interpretações das realidades sociais, procurando descrever os significados dos fenômenos, preocupando-se com crenças, valores e atitudes por tentar atingir a subjetividade dos fatos, sendo abundante em contexto (Pereira, et al., 2018).

No estudo exploratório busca-se melhor entendimento ou a construção de hipóteses acerca do problema. Uma de suas modalidades é o relato de experiência, esse um tipo de estudo em que o pesquisador realiza uma investigação empírica sem um roteiro rígido e que compreende um método abrangente, com a lógica do planejamento, da coleta e da análise de dados (Yin, 2014; Gil, 2017).

O profissional enfermeiro em um instituto de referência em traumatologia e ortopedia como o que foi cenário para este relato, desenvolve assistência especializada junto aos usuários com vistas a minimizar fatores limitantes no período 
perioperatório (como por exemplo, controle da dor, avaliação de risco de trombose venosa profunda e adoção de medidas preventivas, avaliação de risco para desenvolvimento de lesão por pressão e prevenção/tratamento, avaliação de ferida operatória mediante realização de curativos, atendimentos de intercorrências clínicas e seus desdobramentos), orientações e ações educativas dentre outros cuidados e procedimentos inerentes ao contexto hospitalar. Todas estas ações respaldadas pelo Conselho Federal de Enfermagem (COFEN) e guiado pelas diretrizes da Política Nacional de Humanização do Sistema Único de Saúde (Ministério da Saúde do Brasil, 2013).

Para melhor entendimento da experiência de cuidados implementados pelo enfermeiro, dividimos a narrativa em três temas: Fluxo de atendimento e o perfil de pacientes atendidos; Complexidade do atendimento ao paciente com lesão musculoesquelética que vivencia a COVID-19; Novos desafios para a enfermagem ortopédica gerados pela pandemia da COVID-19.

\section{Resultados e Discussão}

\section{Fluxo de atendimento e o perfil de pacientes atendidos}

O indivíduo que sofreu um acidente no estado do Rio de Janeiro e desenvolveu uma lesão musculoesquelética e que necessita de tratamento específico, quer seja clínico ou cirúrgico, comumente procura ou é encaminhado a atendimento em alguma Unidade Básica de Saúde ou um Hospital de Urgência e Emergência. Nos casos em que o tratamento é cirúrgico e a unidade de entrada não disponha, o paciente é encaminhado para o Sistema Estadual de Regulação (SER) do estado do Rio de Janeiro. Ao ser inserido neste sistema o mesmo fica aguardando vaga para alguma unidade especializada que realize o procedimento necessário.

Ao chegar à unidade especializada em Traumatologia e Ortopedia, cenário dessa experiência, o paciente passa por avaliação multiprofissional (médica e da equipe de enfermagem). Caso se constate possível infecção por COVID-19, o mesmo é encaminhado para leitos de isolamento, em enfermarias ou de terapia intensiva, de acordo com sua necessidade.

Em março de 2020, logo no início da pandemia, as cirurgias eletivas desta instituição foram suspensas, sendo realizadas apenas para casos de pacientes com tumores ósseos e de lesões musculoesqueléticas que necessitassem de uma intervenção de curto prazo ou de emergência. A normalização do fluxo de agendamento de cirurgias eletivas teve início em julho de 2020, quando os números de pacientes internados com infecção por vírus SARS- CoV-2 começaram a apresentar redução.

No que se refere ao perfil de pacientes hospitalizados no período em análise, este mudou consideravelmente, sendo representado agora por pacientes encaminhados de outras Unidades de Saúde do Estado do Rio de Janeiro, entre essas Unidades de Pronto Atendimento (UPA), Hospitais Gerais de Urgência e Emergência e Pronto-Socorro. Atendendo o acordo firmado com a Secretaria Estadual de Saúde do Estado do Rio de Janeiro.

A faixa etária e os tipos de lesão variou de acordo com as demandas semanais: entre os jovens a ocorrência era maior de vítimas de acidentes automobilísticos e agressões por conflito violento; já na faixa etária dos idosos destacaram-se as lesões por queda da própria altura e acidente doméstico e re-internações por complicações de pós operatório de pacientes oriundos do próprio instituto relacionadas a infecção do sítio cirúrgico, trombose venosa profunda, falha mecânica entre outras. Além dessas novas admissões, a instituição ainda contava com a assistência aos pacientes com internação de longa permanência que já estavam em tratamento antes do início da pandemia e sem condições clínicas e socioeconômicas de alta hospitalar.

Dentre as populações mais vulneráveis a desenvolver complicações relacionadas à infecção pelo vírus SARS- CoV-2 destacam-se os idosos e os portadores de comorbidades (diabetes, hipertensos, obesidade e doenças respiratórias). Todos com risco moderado a alto de vir a óbito devido às complicações. Dados coletados nos países que enfrentaram a epidemia inicial 
evidenciam que a taxa de mortalidade aumentava conforme se avançava na faixa etária (Zhang, 2020). Aponta-se que este cenário pode ser observado também no Brasil (Monteiro, et al., 2020).

Segundo Bezerra et al (2020), os impactos gerados pela pandemia e suas complicações ocorrem progressivamente com o passar do tempo/idade. $\mathrm{O}$ estresse e o medo passam a ser uma realidade nestes pacientes, fato que determina, em muitos momentos, a ocorrência de dores físicas e psicológicas que contribuem para o surgimento da depressão e ansiedade, influenciando negativamente na qualidade de vida de pessoas acometidas. Em pacientes com lesões musculoesqueléticas, além de enfrentarem o complexo tratamento ortopédico, ainda convivem com as possíveis complicações decorrentes da COVID-19.

\section{Complexidade do atendimento ao paciente com lesão musculoesquelética que vivencia a COVID-19}

O profissional enfermeiro atuou com diferentes e complexas ações neste período pandêmico. Tudo era novo! Tudo deveria ser resolvido no menor espaço de tempo possível. Às ações que já realizava com os pacientes internados com lesões musculoesqueléticas se somaram a outras relacionadas a COVID-19: treinamentos (lavagem das mãos, uso de álcool gel, paramentação / desparamentação, uso de equipamentos, manejo de vias aéreas, transporte de pacientes, entre outros), avaliação do paciente focada nos sintomas respiratórios, hemodinâmicos e vasculares, orientações a familiares, solicitações da necessidade de compras de equipamentos de proteção individual, dentre outras ações. Atuou tanto nos cuidados diretos na perspectiva biopsicossocial e espiritual, como na gestão dos cuidados realizados por outros profissionais.

Durante o atendimento ao paciente, o enfermeiro realiza a Sistematização da Assistência de Enfermagem (SAE) para traçar as melhores condutas no tratamento e na execução dos cuidados. Consiste na aplicação sistematizada e fundamentada por uma teoria de cuidado, das ações de enfermagem desenvolvidas para uma determinada clientela ou população que abrange: Coletar dados acerca do indivíduo, sua coletividade e seu processo saúde-doença; Identificar as deficiências e carências advindas do processo de saúde-doença das informações coletas; Traçar intervenções de forma individualizada com objetivos específicos que visem restaurar o equilíbrio das necessidades humanas afetadas; Avaliar as condutas traçadas conforme os resultado esperados e implementar novas ações, se couber (Tanure \& Pinheiro, 2019).

O paciente ortopédico possui limitações relacionadas às suas lesões musculoesqueléticas, tendo maior propensão a apresentar: mobilidade prejudicada, dor, cinesiofobia, lesões cutâneas, trombose venosa profunda, entre outras que desencadeiam déficit para o autocuidado e consequentemente, auxílio nas atividades de vida diária (Cameron \& Araújo, 2011a). Quando o mesmo é acometido pelo vírus SARS- CoV-2, além das dificuldades inerentes às questões ortopédicas, ele pode apresentar os graves sintomas da COVID-19 e desenvolver complicações como derrame pleural, pneumotórax, pneumonia, fazendo com que precise de um maior suporte para lidar com o tratamento.

Um dos sintomas agravantes é a dispneia persistente com saturação < $95 \%$ que gera a necessidade de oxigenoterapia contínua, dificuldade de locomoção devido à fadiga e fraqueza advindas da infecção, acentuando a necessidade de auxílio de muletas, andador e até mesmo necessidade de ajuda para mobilização no leito. Alguns pacientes precisam de auxílio para realizar as refeições, banho e demais necessidades.

A complexidade assistencial aumenta e são necessários cuidados para pacientes que apresentam alta dependência das ações de enfermagem quanto ao atendimento de suas necessidades humanas básicas. O enfermeiro precisou atuar avaliando as possíveis alterações de estado mental, necessidade contínua de oxigenação, controle de sinais vitais em curtos intervalos, mobilidade limitada, deambulação com auxílio ou restrição ao leito, alimentação por sonda ou com auxílio, cuidados corporais (como higiene oral e banho) bem como auxílio nas eliminações vesicointestinais, além da administração de drogas (vasoativas, corticóides, antibióticos, anticoagulantes), realização de exames (imagem, laboratorial) e outros procedimentos como punções e curativos. 
Todas essas ações de saúde aconteciam sob nova modalidade de atendimento: a precaução de contato e respiratória contínuas. Sendo primordial a oferta de treinamentos para a equipe multiprofissional de saúde sobre: paramentação e desparamentação de equipamentos individuais de proteção; manejo de vias aéreas; transporte seguro de pacientes; identificação precoce de casos suspeitos; preparo do corpo e solicitação frequente de exames. Foi desafiador! Muito aprendizado em curto espaço de tempo.

\section{Novos desafios para a enfermagem ortopédica, gerados pela pandemia da COVID-19}

A enfermagem ortopédica e traumatológica é um campo especializado, relacionada à assistência em situações de doenças, processos congênitos e do desenvolvimento, traumas, infecções, doenças degenerativas e outros comprometimentos que atingem o sistema musculoesquelético, articular e o tecido conjuntivo de suporte. Compreende problemas de saúde clínicos, de reabilitação e cirúrgicos (pré-operatório, transoperatório, recuperação anestésica e pós-operatório) (Cameron \& Araújo, 2011b). Os cuidados de enfermagem transitam de cenários agudos aos crônicos de longa duração e precisam atender eficazmente às especificidades dessas situações, visando a promoção e autonomia através de abordagem integradora (Gomes \& Ferreira, 2010).

Além dos desafios enfrentados durante o atendimento ao paciente ortopédico e as complicações inerentes à infecção por vírus SARS- CoV-2, os profissionais da área da saúde também sofreram com os impactos da pandemia. Segundo o Conselho Federal de Enfermagem o número de profissionais acometidos pela doença aumentou com a propagação do vírus (Soares, 2020). Com o afastamento de alguns profissionais classificados como grupo de risco (portadores de comorbidades e idade superior a 60 anos) e o adoecimento de profissionais infectados pelo vírus, que necessitavam de isolamento e tratamento salutar por no mínimo de 14 dias a assistência ficou diretamente impactada na medida em que o absenteísmo reduzia a oferta de vagas e aumentava a sobrecarga de trabalho.

Durante o atendimento ao paciente internado em enfermarias e acometido pelo vírus SARS- CoV-2 a equipe de enfermagem, liderada pelo enfermeiro, colaborou com as demandas assistenciais através da avaliação e comunicação das alterações do quadro de saúde do paciente à equipe multidisciplinar (médica - clínicos, ortopedistas e infectologistas; psicologia, nutrição, serviço social). Durante a passagem de plantão a equipe de enfermagem discutia sobre a performance biopsicossocial de cada paciente e suas necessidades eram levadas diretamente aos membros da equipe multidisciplinar, bem como à enfermeira rotina responsável pelo setor, promovendo comunicação e buscando resolução dos problemas diagnosticados.

No período de isolamento social para o tratamento hospitalar o paciente é privado de receber visitas ou de ter um acompanhante, com isso muitos pacientes relatam medos e angústias devido às incertezas referentes ao seu futuro e de seus entes queridos. Alguns deles não possuíam telefone celular para contato com a família, seja por dificuldades financeiras ou mesmo por falta de manejo tecnológico.

Em alguns casos, os profissionais auxiliavam os pacientes com dificuldades para uso com o celular ou através de solicitação ao Serviço Social eram repassadas informações aos familiares sobre as necessidades de artigos de higiene pessoal (xampu, condicionador, hidratantes), roupas (íntimas, meias e blusa de frio), óculos, chinelos, prótese dentária e outros produtos permitidos na enfermaria como: livros, mini-rádio e aparelhos de celulares.

Quando avaliado a necessidade de acompanhamento psicológico, o enfermeiro comunicava a equipe de Saúde Mental solicitando o atendimento, sendo este realizado de maneira remota quando o paciente possuía um aparelho celular ou presencialmente, de acordo com as demandas. 
Ao avaliar as dificuldades de ingestão alimentar, por exemplo, o nutricionista era acionado pelo enfermeiro junto ao Serviço de Nutrição. Demandas eram discutidas em conjunto com a equipe médica em busca de atendimento das particularidades.

Diante desse cenário o enfermeiro, através da troca de mensagens verbais e não-verbais, por meio da fala, bilhetes, por um gesto ou de um olhar afetuoso e acolhedor, estabeleceu vínculos afetivos com pacientes, que se tornaram importantíssimos para o enfrentamento da gravidade imposta pela Pandemia da COVID-19.

Com as experiências geradas durante a Pandemia a equipe de enfermagem se deparou com grandes desafios relacionados à complexidade das demandas dos pacientes e ao contexto de incertezas sobre o vírus e suas repercussões futuras, assim estes momentos foram de riquíssimo aprendizado não só sobre a questões relacionadas à assistência, mas também a forma como os profissionais estavam inseridos no processo apoiando uns aos outros.

\section{Considerações Finais}

Este relato de experiência trata dos cuidados do enfermeiro diante de situações desafiadoras de infecção por COVID19 em pacientes com lesões musculoesqueléticas em pré-operatório e/ou submetidos a cirurgia ortopédica. Os achados podem ser replicados em outros contextos similares em que acolhem-se e assistem-se pacientes ortopédicos que vivenciam a COVID19 por trazer encaminhamentos e sugestões de enfrentamento para as demandas geradas por esta pandemia.

A atuação do enfermeiro com competência técnica, autônoma e na perspectiva da integralidade do cuidado durante o processo terapêutico do paciente ortopédico acometido pelo Coronavírus, contribui para o atendimento das singularidades, evidenciando a importância deste profissional. Ao adotar estratégias inovadoras no cuidado, este profissional promove a melhoria da qualidade assistencial já que abrange não só o paciente, mas sua família e a equipe de saúde por meio de ações de educação em saúde que promovem o desenvolvimento da cidadania e consciência sanitária.

O enfrentamento à COVID-19 deve ser organizado pela articulação de ações em todas as dimensões múltiplas afetadas, dentre as quais os mecanismos imunológicos envolvidos, já que a velocidade do adoecimento e a gravidade são maiores e mais intensas do que a capacidade de atendimento dos serviços de saúde (Guimarães, 2020). A descoberta de um produto seguro e eficaz para prevenir as infecções - VACINA - é a esperança para deter o avanço da doença que vitimou milhões de pessoas no mundo (Stevanim, 2020).

Segundo a Organização Mundial da Saúde, atualmente, há 30 projetos em pesquisa clínica e outros 139 em avaliação pré-clínica. Nove desses na última fase de testes em humanos (fase 3), sendo dois com acordos para serem produzidos no Brasil - Universidade de Oxford e AstraZeneca em parceria com a Fiocruz e Sinovac, na China, com o Instituto Butantan (Stevanim, 2020). O Brasil iniciou a campanha de vacinação no dia 17 de janeiro de 2021 com a liberação da Agência Nacional de Vigilância Sanitária (ANVISA) para ambas as parcerias e há outras em fase de autorização para o consumo no país.

Em 2021, a pandemia continua impactando a vida de populações e dos profissionais de saúde de diversas maneiras. Apesar dos esforços dos serviços de saúde e de todos os profissionais que atuam na linha de frente, o número de mortos ainda se mantém elevado e o perfil dos afetados vem sendo alterado cotidianamente. Os estudos existentes até o momento não mostraram benefício com o tratamento precoce e alguns destes medicamentos podem causar efeitos colaterais, sendo não recomendado pela Sociedade Brasileira de Infectologia (SBI), que está alinhada com as recomendações da Sociedade de Infectologia dos EUA (IDSA) e Europa (ESCMID), o Centro Norte-Americano de Controle e Prevenção de Doenças (CDC), a Organização Mundial da Saúde (OMS) e a Agência Nacional de Vigilância Sanitária do Ministério da Saúde do Brasil (ANVISA). O que há de mais promissor é a busca pela vacina e a manutenção das medidas de proteção e distanciamento social (SBI, 2020). 
Durante a pandemia, o enfermeiro vivenciou uma nova realidade de assistência, experimentando e produzindo conhecimento junto ao paciente ortopédico com COVID-19 e a equipe multidisciplinar no que concerne a avaliação, diagnóstico e ações direcionadas às demandas biopsicossociais, tecnológicas, estruturais e afetivas, que ainda representam um grande desafio. Na produção deste relato de experiência foram encontradas limitações relacionadas a achados na literatura sobre assistência de pacientes ortopédicos acometidos pela COVID-19 e os desdobramentos gerados pelo atendimento. Recomenda-se a realização de novos estudos sobre a temática para fins de análises comparativas e a ampliação do conhecimento nesta área.

\section{Agradecimentos}

Agradecemos a todos os pacientes atendidos durante a pandemia e seus familiares, a equipe multiprofissional envolvida na assistência da instituição hospitalar onde ocorreu o estudo, ao Programa Acadêmico em Ciências do Cuidado da Universidade Federal Fluminense e a Coordenação de Aperfeiçoamento de Pessoal de Nível Superior - CAPES.

\section{Referências}

Bezerra, D. R. C., Paulino, E. T., Santo, F. H. E., Magalhães, R. S., Silva. (2020). Uso das Práticas Integrativas e Complementares no período de isolamento social da COVID-19 no Brasil. Research, Society and Development, 9(11), 13-29. https://rsdjournal.org/index.php/rsd/article/view/9718

Cameron, L. E., \& Araújo, S. T. C. (2011a) O estudante de graduação e a assistência em enfermagem traumato-ortopédica. Rev. Latino-Am. Enfermagem, 19(6), 1391-1397. https://www.scielo.br/pdf/rlae/v19n6/pt_16.pdf

Cameron, L. E. \& Araújo, S. T. C. (2011b). Vision as an instrument of perception in trauma and orthopedic nursing care. RevEscEnferm USP, 45(1), 95- 9. http://www.scielo.br/pdf/reeusp/v45n1/en_13.pdf.

Francisco Neto, M. J., \& Queiroz, M. R. G. de. (2020). Uso racional da ultrassonografia de tórax no enfrentamento da COVID-19. Radiologia Brasileira, 53(5). https://doi.org/10.1590/0100-3984.2020.53.5e3

Gil, A. C. (2017). Como elaborar projetos e pesquisa. (6a ed.) Atlas

Gomes, B. P. \& Ferreira, M. R. S. (2010). Assistência de enfermagem em ortopedia e traumatologia. Enferm. Glob. Murcia, 20 (10). http://scielo.isciii.es/scielo.php?script=sci_arttext\&pid=S169561412010000300025\&lng=es\&nrm=iso.

Guimarães, R. (2020). Vacinas Anticovid: um Olhar da Saúde Coletiva. Ciência \& Saúde Coletiva. 25 (9), 3579-3585. https://doi.org/10.1590/141381232020259.24542020

Ministério da Saúde do Brasil, Secretaria de Atenção à Saúde (2013). Política Nacional de Humanização. Humaniza SUS. Ministério da Saúde.

Ministério da Saúde do Brasil. (2020). Protocolo de manejo clínico para o novo-Coronavírus https://portalarquivos2.saude.gov.br/images /pdf/2020/fevereiro/11/protocolo-manejo- -coronavirus.pdf.

Monteiro, J. K. M.F., Sá, S. P.C., Bezerra, D. R. C., Borges, W. D. (2020). Recomendações aos cuidadores e familiares de idosos mediante o COVID-19. Research, Society and Development, 9(11), 1-16. http://dx.doi.org/10.33448/rsd-v9i11.9798

O'Dwyer, G. O., Oliveira, S. P. \& Seta, M. H. (2009). Avaliação dos serviços hospitalares de emergência do programa QualiSUS. Ciência \& Saúde Coletiva, 14(5), 1881-1890. https://dx.doi.org/10.1590/S1413-81232009000500030

Pereira A. S. et al. (2018). Metodologia da pesquisa científica. UFSM. https://repositorio.ufsm.br/bitstream/handle/1/15824/Lic_Computacao_MetodologiaPesquisa-Cientifica.pdf?sequence $=1$

Rubin G. D., Ryerson C. J., Haramati L. B., et al. (2020). The role of chest imaging in patient management during the COVID-19 pandemic: a multinational consensus statement from the Fleischner Society. Radiology. 296, 172-80.

Soares, F. (2020). Pandemia COVID-19: Formação e Atuação da Enfermagem Para o Sistema Único de Saúde. Enferm. Foco, 11 (1), 40-45. http://biblioteca.cofen.gov.br/pandemia-COVID-19-formacao-atuacao-enfermagem-sus/

Sociedade Brasileira de Infectologia [SBI]. (2020). Atualizações e recomendações sobre a COVID-19. https://infectologia.org.br/wpcontent/uploads/2020/12/atualizacoes-e-recomendacoes-COVID-19.pdf

Stevanim, L. F. (2020). Uma vacina para a humanidade: da expectativa à realidade, os esforços para se chegar a uma vacina contra COVID-19 acessível à população. RADIS: Comunicação e Saúde. 216 (6), 12-21. https://www.arca.fiocruz.br/handle/icict/43683.

Tannure, M. C. \& Pinheiro, A. M. (2019) SAE: Sistematização da Assistência de Enfermagem: Guia Prático. (3a ed.). Guanabara Koogan. 
Research, Society and Development, v. 10, n. 3, e25110312947, 2021

(CC BY 4.0) | ISSN 2525-3409 | DOI: http://dx.doi.org/10.33448/rsd-v10i3.12947

The World Bank. (2014). Global Road Safety Facility; University of Washington. Institute for Health Metrics and Evaluation. Transport for health: the global burden of disease from motorized road transport. Seattle, http://documents.worldbank.org/curated/pt/9842614683270021 20/pdf/863040IHME0T4H0ORLD0BANK0compressed.pdf.

World Health Organization [WHO]. (2020) Director-General's opening remarks at the media briefing on COVID-19 - March 11, 2020. https://www.who.int/dg/speeches/ detail/who-director-general-s-opening-remarks-at-the-media-briefing-on-COVID-19-11-march-2020

Yin, R. K. (2014). Estudo de caso: planejamento e métodos. (5a ed.). Bookman.

Zhang, W. (2020). Manual de Prevenção e Controle da COVID-19 segundo o Doutor Wenhong Zhang. PoloBooks. 